\title{
TALEN-mediated apc mutation in Xenopus tropicalis phenocopies familial adenomatous polyposis
}

\author{
Tom Van Nieuwenhuysen ${ }^{1}$, Thomas Naert ${ }^{1}$, Hong Thi Tran ${ }^{1}$, Griet Van Imschoot ${ }^{1}$, \\ Sarah Geurs ${ }^{1}$, Ellen Sanders ${ }^{2,3}$, David Creytens $^{4}$, Frans Van Roy ${ }^{2,3}$ and Kris \\ Vleminck ${ }^{1,5}$ \\ ${ }^{1}$ Developmental Biology Unit, Department of Biomedical Molecular Biology, Ghent University, Ghent, Belgium \\ ${ }^{2}$ Molecular Cell Biology Unit, Department of Biomedical Molecular Biology, Ghent University, Ghent, Belgium \\ ${ }^{3}$ Inflammation Research Center, Flanders Institute for Biotechnology (VIB), Ghent, Belgium \\ ${ }^{4}$ Department of Pathology, Ghent University and Ghent University Hospital, Ghent, Belgium \\ ${ }^{5}$ Center for Medical Genetics, Ghent University and Ghent University Hospital, Ghent, Belgium \\ Correspondence to: Kris Vleminckx, email: Kris.Vleminckx@irc.UGent.be \\ Keywords: APC, Intestinal cancer, desmoid tumors, Wnt signaling, animal model \\ Received: April 8, $2015 \quad$ Accepted: May 17, $2015 \quad$ Published: May 19, 2015
}

This is an open-access article distributed under the terms of the Creative Commons Attribution License, which permits unrestricted use, distribution, and reproduction in any medium, provided the original author and source are credited.

\section{ABSTRACT}

Truncating mutations in the tumor suppressor gene adenomatous polyposis coli (APC) are the initiating step in the vast majority of sporadic colorectal cancers, and they underlie familial adenomatous polyposis (FAP) syndromes. Modeling of APCdriven tumor formation in the mouse has contributed substantially to our mechanistic understanding of the associated disease, but additional models are needed to explore therapeutic opportunities and overcome current limitations of mouse models. We report on a novel and penetrant genetic cancer model in Xenopus tropicalis, an aquatic tetrapod vertebrate with external development, diploid genome and short life cycle. Tadpoles and froglets derived from embryos injected with TAL effector nucleases targeting the apc gene rapidly developed intestinal hyperplasia and other neoplasms observed in FAP patients, including desmoid tumors and medulloblastomas. Bi-allelic apc mutations causing frame shifts were detected in the tumors, which displayed activation of the $\mathbf{W n t} / \beta$-catenin pathway and showed increased cellular proliferation. We further demonstrate that simultaneous double bi-allelic mutation of apc and a non-relevant gene is possible in the neoplasias, opening the door for identification and characterization of effector or modifier genes in tumors expressing truncated apc. Our results demonstrate the power of modeling human cancer in Xenopus tropicalis using mosaic TALEN-mediated bi-allelic gene disruption.

\section{INTRODUCTION}

FAP is an autosomal dominant disorder characterized by the presence of hundreds to thousands of benign, adenomatous polyps in the colon, which over time can progress to malignant adenocarcinomas. FAP is caused by germline mutations in the $A P C$ gene. Different variants of FAP exist. In the Gardner syndrome (OMIM $175100,135290)$, gastrointestinal adenoma formation is frequently accompanied by extra-colonic manifestations, such as congenital hypertrophy of the retinal pigment epithelium (CHRPE), desmoid tumors, osteomas, dental anomalies, epidermal cysts, and soft tissue tumors. In patients diagnosed with the Turcot syndrome (OMIM 276300), central nervous system malignancies, such as medulloblastomas, are also observed. Although prophylactic colorectal surgery significantly reduces the mortality associated with FAP, the other less penetrant signs and symptoms are becoming more clinically relevant, most prominently the desmoid tumors, which are hard to treat and are a major cause of death in FAP patients $[1,2]$. 
Nonsense mutations in the $A P C$ gene underlie FAP, and these mutations are also observed in most sporadic colon cancers. Several mouse FAP models have been identified or generated [3], the most often used model being the Apc ${ }^{\mathrm{Min}}$ mouse. Conditional models with floxed Apc alleles have also been generated [3]. Recently intestinal tumor formation has also been described in rats and zebrafish carrying ENU-induced mutant $A p c$ alleles $[4,5]$.

Mutations in the $A P C$ gene result in the expression of a truncated APC protein that causes ectopic constitutive activation of the Wnt/ $\beta$-catenin pathway, which is seen as an important target for therapeutic intervention. Unfortunately, the Wnt pathway is notoriously difficult to target by small chemical compounds [6, 7]. Many screening efforts have so far not yielded any applicable drug urging the need for alternative animal models such as Xenopus that allow fast and reliable screening of novel therapeutics or potential therapeutic targets $[8,9]$.

Modeling human disease in non-mammalian vertebrates has been primarily restricted to zebrafish, which has been subjected to large-scale mutagenesis screens $[10,11]$. Therefore, zebrafish is being increasingly used as a cancer model [12]. Until now, Xenopus has not reached the same status because it has been used mostly for transient approaches such as RNA and Morpholino injections. However, TALEN and CRISPR/Cas9 mediated genome editing is creating a revolution in the field of functional genomics [13], opening the door for many novel experimental approaches and strategies in numerous model organisms. It is easy and economical to make custom TALEN and CRISPR/Cas9 constructs, and the technologies are within reach of any laboratory
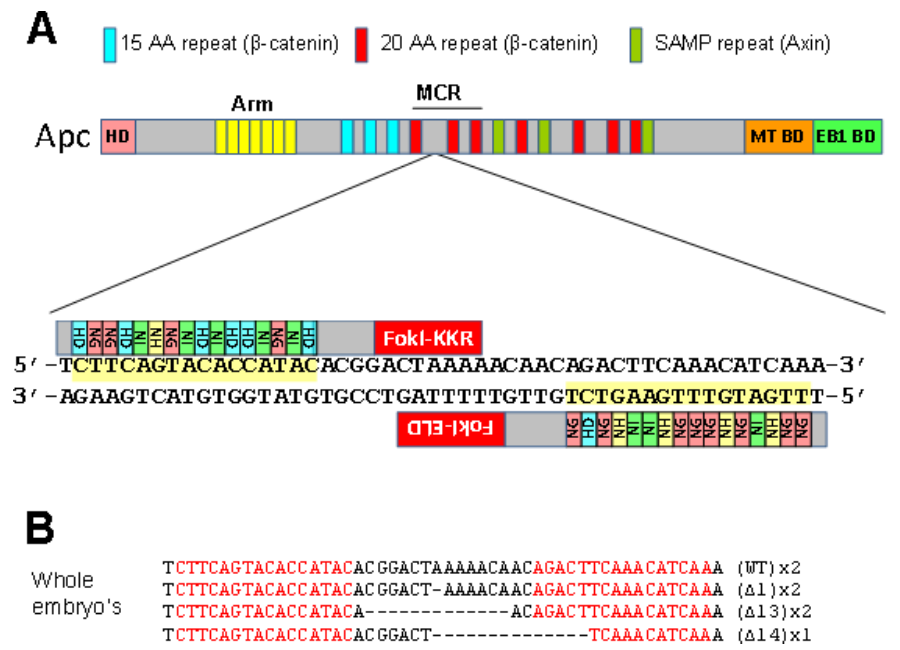

with standard molecular biology facilities. Moreover, zebrafish and Xenopus are very suitable for knockout experiments because they develop externally and require simple injection setups, thus allowing cheap semi-highthroughput gene knock-out approaches. Importantly, unlike $X$. laevis and zebrafish, $X$. tropicalis has a true diploid genome and can therefore gain in importance for modeling human diseases, including cancer [14].

Here, we describe the first genetic cancer model in $X$. tropicalis. Using TALEN-mediated targeting of the mutation cluster region (MCR) of the apc allele, we developed a model that mimics the human FAP syndrome and remarkably recapitulates several of the tumor types observed in FAP patients. This model may pave the way for establishing other cancer models in X. tropicalis and for its use in pre-clinical drug screening.

\section{RESULTS}

\section{Design and use of a TALEN construct targeting the apc MCR in Xenopus tropicalis}

We first determined the domain structures of the $X$. tropicalis apc gene and its encoded protein. The structural and the functional domains of the frog APC protein are very similar to their human counterparts, including a potential N-terminal homodimerization domain, an armadillo repeat region, three repeats of 15 amino acids (aa), seven 20-aa repeats responsible for binding $\beta$-catenin, three SAMP repeats known to interact with axin, a microtubule-binding domain, and a C-terminal EB-1 binding region (Fig. 1A) [15]. Importantly, as in

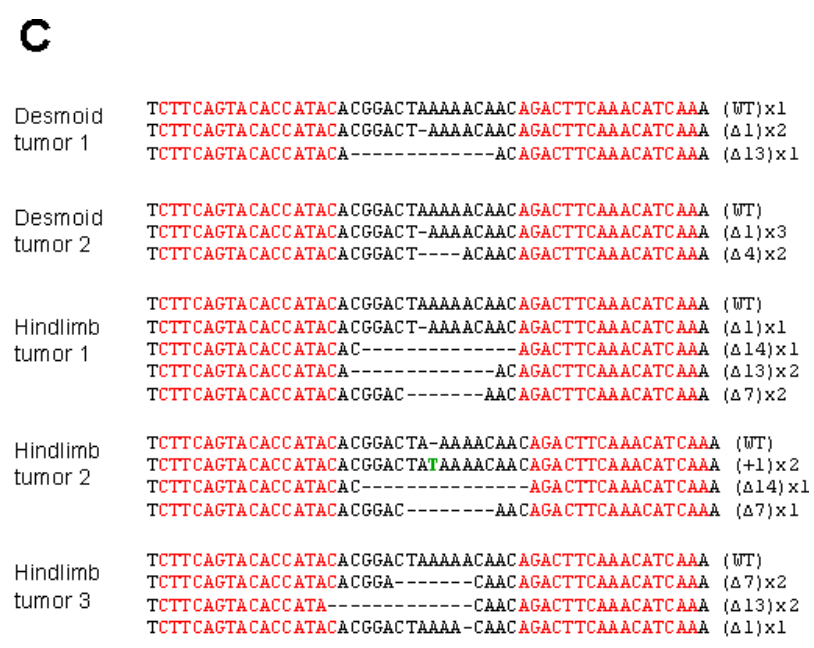

Figure 1: TALEN mediated targeting of the MCR of the Xenopus tropicalis apc gene. (A) Schematic representation of the apc protein, the region that was targeted by TALENs, and the TALENs used. (B) Deletions detected in the targeted region of apc gene in embryos injected with $300 \mathrm{pg}$ of apc TALEN mRNA. The sequences in red indicate the apc TALEN recognition sites. (C) Bi-allelic mutation of the $a p c$ gene identified in desmoid and hindlimb tumors dissected from tadpoles having undergone apc TALEN mRNA (40 pg) injection. AA, Amino Acid; APC, Adenomatous Polyposis Coli; Arm, Armadillo repeats; MCR, Mutation Cluster Region; EB1 BD, End Binding protein 1 Binding Domain; HD, Homodimerization domain; MT BD, Microtubule Binding Domain. 
mammals, the last exon of the apc gene is very long, encoding three-fourths of the protein. Therefore, nonsense or frame-shift mutations in the sequence downstream of the armadillo repeats escape nonsense-mediated decay, and so can generate truncated proteins.

In humans, most truncating $A P C$ mutations occur between the first and third 20-aa repeats [15]. We first sequenced this region from four frogs in our colony to detect any single nucleotide polymorphisms (SNPs) that might interfere with TALEN binding, but none were found. We then designed a TALEN construct to target a sequence between the first and the second 20-aa repeats. An apc TALEN pair was assembled using Golden Gate cloning [16]. Each TALEN arm was fused to either ELD or KKR mutant Fok1, which is active as an obligate heterodimer, hence increasing specificity [17]. No off-target sites were predicted by the TALEN-targeter software [18].

Synthetic RNAs generated from the apc TALEN pair were micro-injected into two-cell stage embryos, which were then grown until stage 46. Individual embryos were lysed and genomic DNA was extracted. The targeted region was amplified and subcloned into pTOPO plasmids. Sequencing of individual plasmid clones revealed discrete insertion and deletion mutations (INDELs) in 59\% $(\mathrm{n}=$ 17) of the clones (Fig. 1B). This demonstrates that the apc TALEN strategy is very efficient in $X$. tropicalis allowing introduction of frame-shifting INDELs in the apc MCR region. These mutations are predicted to result in expression of truncated proteins, which mimic the human disease-prone situation.

\section{Formation of tumors with bi-allelic apc mutation}

High doses (300 pg) of apc TALEN were largely detrimental to embryonic development, most likely because they disrupt several early developmental processes that are under control of $\mathrm{Wnt} / \beta$-catenin signaling. However, a large portion of embryos injected with lower doses $(40 \mathrm{pg}$ ) progressed into the tadpole stage. When the tadpoles developed their hind limbs, several external neoplastic lesions developed. Most striking were the many cyst-like tumors in the epidermis, several subcutaneous tumors, and large, fast-growing tumors in the region of the extending limbs.

APC-driven intestinal tumor formation in humans is associated with bi-allelic mutations or loss of the wild type copy evidenced by loss of heterozygosity [19]. So we analyzed whether the tumors appearing in the tadpoles injected as an embryo with apc TALENS had two mutant apc alleles. Indeed, in each of the five subcutaneous and hind limb tumors that we analyzed, at least two different INDEL mutations in the apc MCR were apparent (Fig. 1C). All INDELs observed induced frame-shifts (e.g. $+1, \Delta 4, \Delta 14)$. This is in line with the expectation that a subpopulation of the embryonic cells will develop truncating bi-allelic mutations that enable them to hyperproliferate and form tumors.
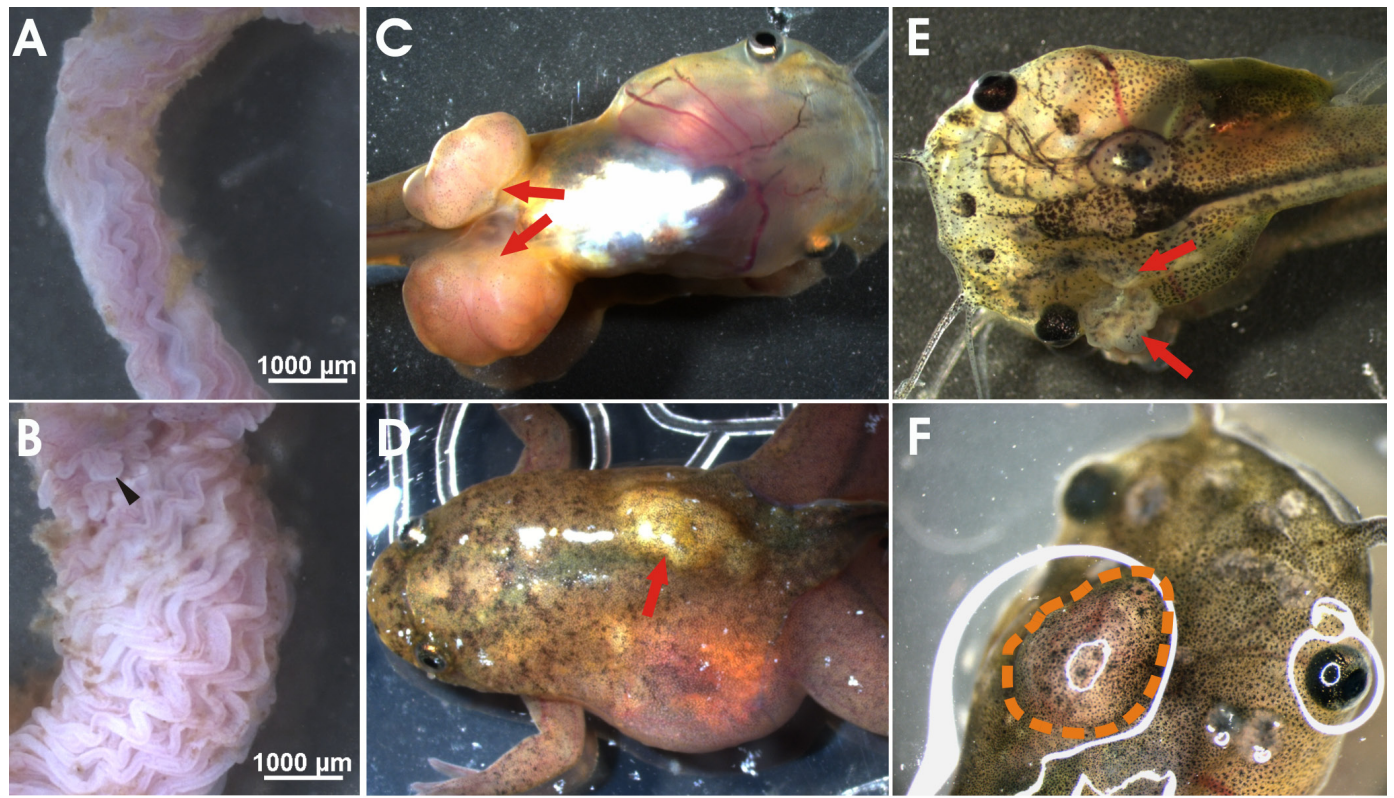

Figure 2: Neoplastic phenotypes observed in apc TALEN mRNA injected tadpoles and frogs. Small intestine, cut open longitudinally, of wild type (WT) (A) and apc TALEN mRNA (40 pg) injected (B) adult frogs. In WT frogs, the epithelial lining of the duodenum is organized in parallel longitudinal folds. The intestines of apc TALEN injected animals are largely expanded and the intestinal folds are irregular and excessively undulated. Aberrant local protuberances are visible (black arrowhead). (C) Large fast growing tumors at the position where the hindlimbs emerge (red arrows). (D) Desmoid tumor, visible as a subcutaneous light colored mass (red arrow). (E) Epidermoid cysts (red arrows) originating at various positions in the tadpole skin. (F) Medulloblastoma visible as a large mass in the tadpole brain region. 
Table 1: Overview of the externally visible tumor phenotypes in animals injected at the 8-cell stage with $20 \mathrm{pg}$ of apc TALEN mRNA in a single blastomere.

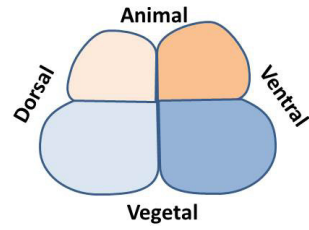

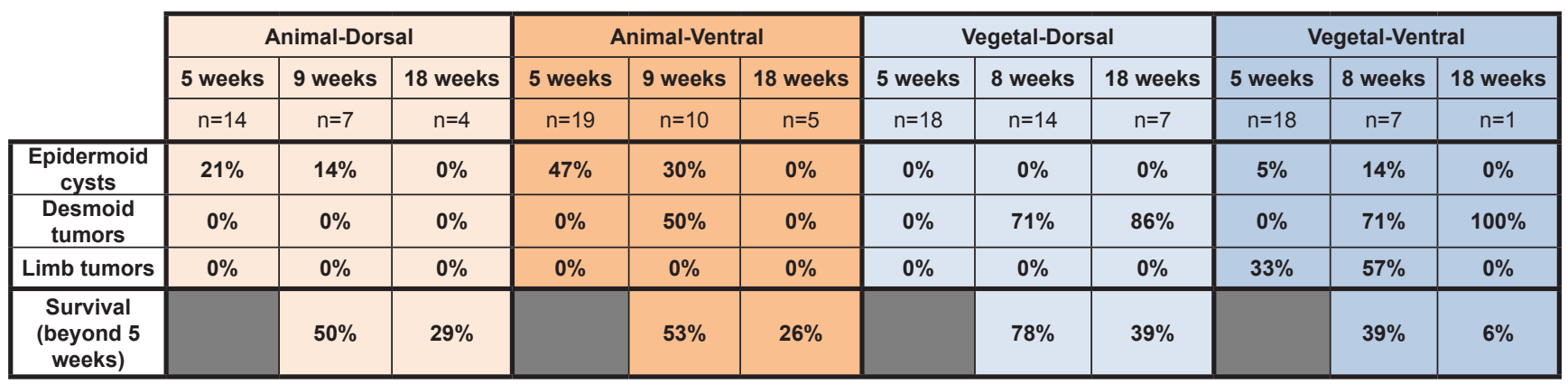

\section{Intestinal and extra-intestinal hyperplasia and malformations in mosaic apc mutant tadpoles and froglets}

The mosaic apc mutant tadpoles and froglets rapidly developed externally visible neoplastic lesions. Most recurrent were subcutaneous desmoid tumors (55\%, $\mathrm{n}=26$ ), epidermoid cysts, fast growing tumors in the limb region $(32 \%)$ and visible abnormalities in the brain $(68 \%)$ and the eyes. The animals were euthanized when they showed clear signs of discomfort. This was especially the case for animals with the limb tumors, which grew fast to a very large size. This prevented the tadpoles form reaching metamorphosis. Hence, to increase tadpole survival, we performed targeted injections of the apc TALEN RNAs in unique individual blastomeres at the 8-cell stage. Xenopus embryos have well characterized fate maps, which allows to enrich the injected substances, such as the TALEN RNAs, in specific tissues and organs [20]. Embryos were injected in a single dorsal-animal, ventral-animal, dorsalvegetal or a ventral-vegetal blastomere and the resulting tadpoles were observed for over 18 weeks. Interestingly, different tumor types were clearly linked to specific injection sites (Table 1). Epidermoid cysts were especially observed in embryos targeted in a ventral-animal or a dorsal-animal blastomere, which contribute primarily to ectodermal derived tissues such as the epidermis. In contrast, desmoid tumors were only observed upon injection of a dorsal-vegetal or ventral-vegetal blastomere,
WT

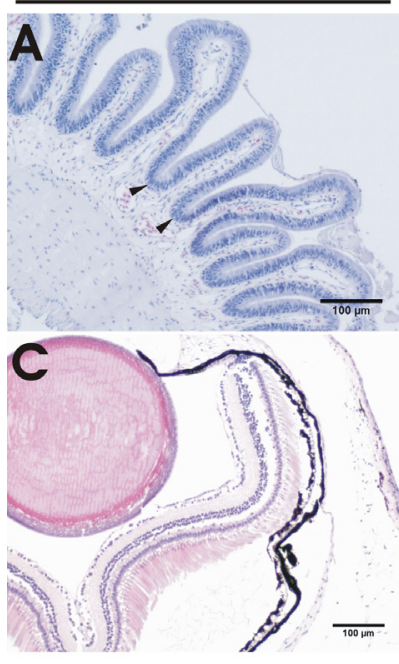

Figure 3: Histological analysis of intestinal and retinal sections. (A) Haematoxylin and eosin (H\&E) staining on a transversal section of the duodenum of a wild type frog. The mucosa is organized in regular alternating long and short folds and troughs (black arrowheads). (B) The small intestine of apc TALEN mRNA (40 pg) injected frogs shows irregular, branched folds (red arrowheads) and expanded troughs (black arrowheads). (C) Cross section through the eye of a WT tadpole showing the lens and the spatially organized retinal layers. (D) The eye of an apc TALEN mRNA (40 pg) injected tadpole displays hyperproliferation and abnormal layering of the neural retina showing rosette-like structures (black arrowheads) and ectopically localized retinal pigment epithelium (red arrowheads). In addition lens differentiation is affected. 
which contribute to the endodermal and mesodermal derived tissues and organs. The fast growing limb tumors were restricted to embryos injected in a ventralvegetal blastomere, which contributes to the lateral plate mesoderm, from which the limb buds originate. Therefore, subsequent exclusion of injections in the ventral-vegetal region can prevent the fast growing limb tumors and hence increase the number of animals that reach metamorphosis.

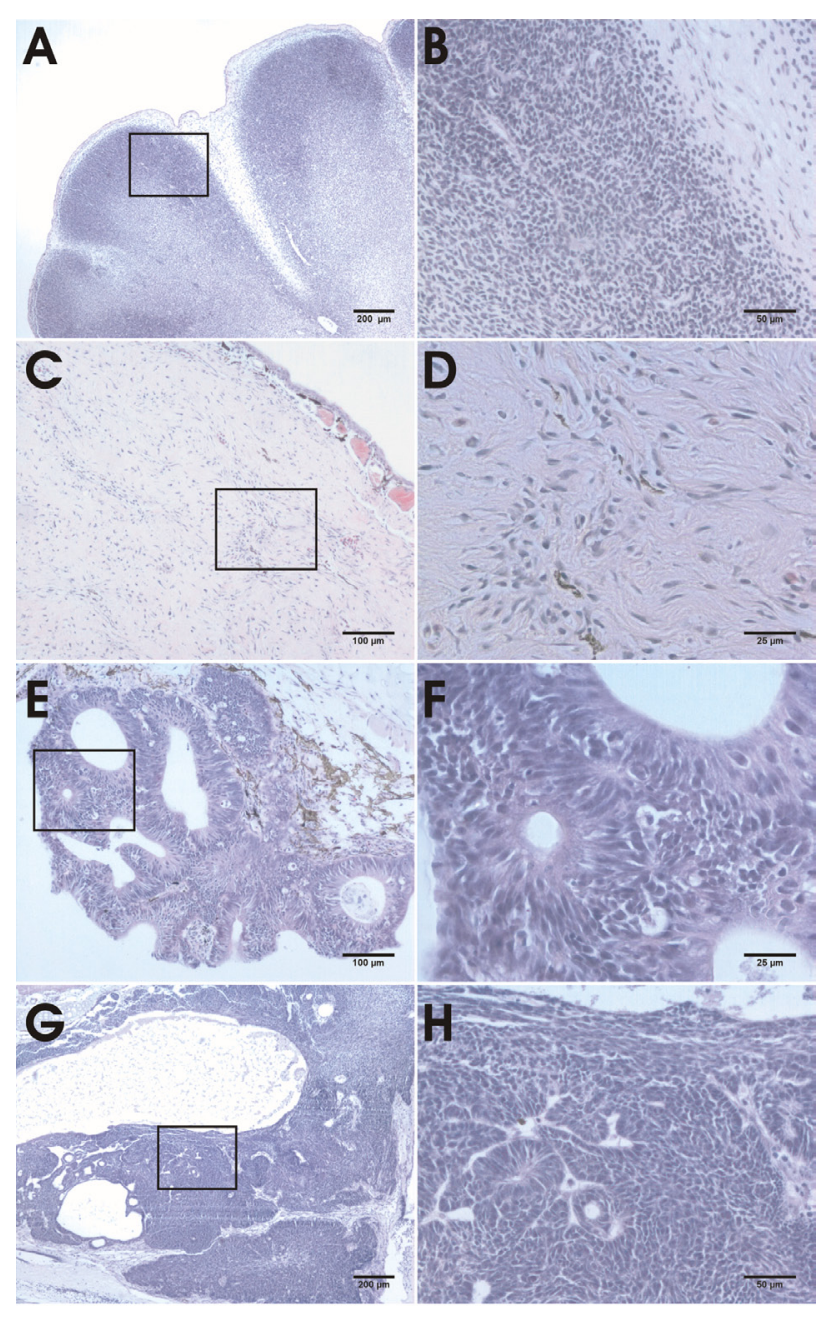

Figure 4: Histological analysis of hind limb, desmoid, epidermal and brain neoplasias of apc TALEN mRNA $(40 \mathrm{pg})$ injected tadpoles and froglets. The boxed regions in the left panels are shown at higher magnifications in the corresponding panels on the right. (A, B) Tumors associated with the developing hind limb are composed of uniform sheets of densely packed monotonously small, round cells with scant cytoplasm. (C, D) Desmoid tumor composed of spindle shaped or stellate fibroblastic cells interspersed with collagen deposits and with prominent vasculature. (E, F) Epidermoid cyst with neuroblastic elements, morphologically reminiscent of a malignant, poorly differentiated, primitive, embryonal neuroepithelial/neuroectodermal tumor (PNET). (G, H) Brain tumor with the histological characteristics of a medulloblastoma.

\section{Intestinal neoplasms}

Intestinal organs were examined after opening of the peritoneal cavity. The liver was often enlarged and yellowish, but there was no evidence of tumor nodules (data not shown). The pancreas, kidneys and spleen did not show obvious macroscopic abnormalities. Although the small and large intestines in tadpoles did not show external signs of tumors, histological examination revealed abnormal organization of the intestine in tadpole stages (in two out of three intestines analyzed - data not shown). In adult frogs dissected at 10 months of age we observed local expansion of the intestine, especially in the duodenum (5 out of 15 intestines analyzed). This was especially apparent when the intestine was cut along the longitudinal axis and turned inside-out. The post-metamorphic intestinal cell layers are organized in longitudinal folds, and the trough-crest axis resembles the crypt-villus organization of mammalian small intestine. Lgr5-positive stem cells are present at the bottom of the troughs, again resembling the situation in mammals [21]. In contrast to wild-type frogs, the folds in the duodenum of animals injected with apc TALENs were massively expanded, irregularly structured and more undulated with local protuberances (Fig. 2A,B).

Histological examination of the intestine of $a p c$ TALEN injected post-metamorphic frogs revealed several abnormalities. Cross-sections of the normal intestine showed a thick muscularis externa lined by a mucosa evaginating into the lumen and forming multiple folds (Fig. 3A). These folds were tall and regular, with alternating long and shorter projections. The folds were lined by a monolayer of tall columnar cells overlying a lamina propria, which was in direct contact with the muscularis externa. The epithelial cells at the base and between the folds had a larger, darker stained elongated nucleus in a basal location. Towards the tip of the folds the nuclei were smaller and mucus-secreting goblet cells were present between the enterocytes.

Transversal sections of the intestine from the mutated animals showed expanded crypts and folds that were very irregular and of various length (Fig. 3B). Several folds showed ectopic crypts perpendicular to their longitudinal axis. The epithelial cells lining the cavity were small, but tall columnar cells with an elongated basal nucleus were present from the top to bottom of the folds and the number of cells lining the folds was increased relative to the normal samples. Features of inflammation were not observed in either normal or mutated samples.

\section{Limb associated tumors}

Rapidly growing tumors with external lobular morphology appeared in the hind limb region of $30 \%$ of tadpoles injected at the 2-cell stage, often displacing the 
developing limb (Fig. 2C). A higher incidence ( $>70 \%$ ) of limb tumors (including tumors in the front limb region) was observed upon targeted injection of a ventral-vegetal blastomere (Table 1). These tumors were sometimes very large, with the inward growing part displacing the organs in the abdomen. Histological examination showed tumors with a solid and relatively circumscribed growth arrangement composed of patternless sheets of densely packed, monotonously small, round, cells with scant cytoplasm (SRBCT or small round blue cell tumors) (Fig. 4A,B). The most cell-dense areas were at the periphery. The central regions were more scarcely populated and contacted the forming limb structures. There was no discontinuity between the limb tissue and the neoplasm. This indicates that the tumor might have been derived from the limb mesenchyme.

\section{Desmoid tumors}

A tumor type observed frequently in more than $30 \%$ of tadpoles injected at the 2-cell stage with apc TALENs was externally visible as subcutaneous light-colored masses (Fig. 2D). Tumor frequency surpassed $70 \%$ when the TALENs were injected at the 8-cell stage in a vegetal blastomere (Table 1). These tumors were relatively slow growing and became detectable in premetamorphic tadpoles (Fig. 2D). They were mostly attached to skin and/or muscles. Histology revealed a spindle-cell mesenchymal tumor composed of sweeping fascicles of uniform, spindle shaped or stellate, fibroblastic cells interspersed with collagen deposits and with a prominent vasculature (Fig. 4C,D). These features are characteristic of desmoid fibromatosis, a major extra-colonic tumor prevalent in FAP patients [22].

\section{Epidermoid cysts}

The first tumors that were apparent in developing tadpoles were epidermal neoplasms, mostly detectable from two weeks of age on (Fig. 2E). The highest frequency $(47 \%)$ was observed when targeting the TALENs to an animal-ventral blastomere, which contributes to the epidermis (Table 1). Histological examination revealed a cystic organization, also suggesting an epidermal origin (Fig. 4E). Although epidermoid cysts have been observed in FAP patients [22], their histological architecture is very different from the frog tumors. This may be due to differences in the organization of the skin, which in Xenopus tadpoles is not stratified. The cystic outgrowths contained neuroblastic elements with formation of rosettes, morphologically reminiscent of a malignant, poorly differentiated, primitive, embryonal
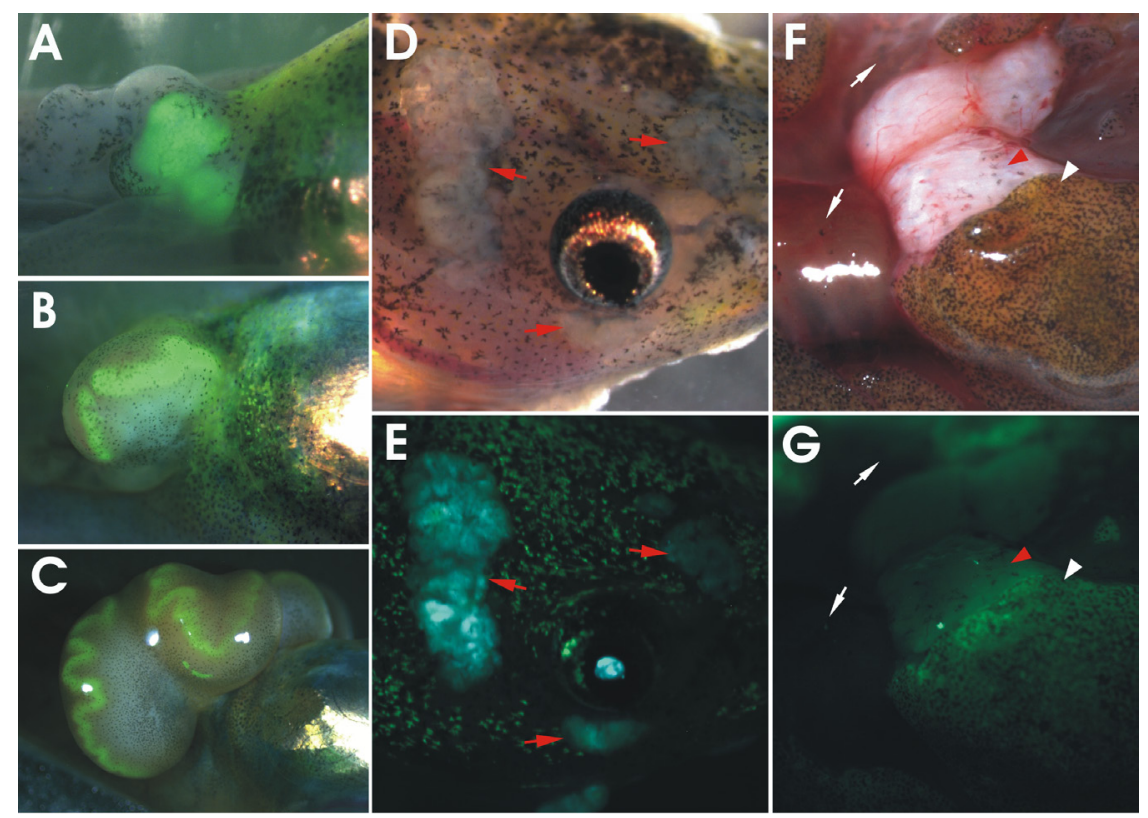

Figure 5: Active Wnt signalling in apc TALEN induced tumors. GFP expression is increased in tumors developing in apc TALEN treated embryos derived from a transgenic Wnt reporter line [23]. (A-C) Intense GFP expression reflecting Wnt activity in a hind limb associated tumor. The same tumor is shown 3, 4 and 7 weeks after apc TALEN injection. Active Wnt signalling is detected in the entire tumor in the initial stages (A) but gets restricted to undulated strands at the edge of the tumor in the later stages (B, C). (D) Brightfield and (E) fluorescent image of epidermoid cysts (red arrows) showing active Wnt signalling. Fluorescence of the neighboring skin is due to autofluorescence. (F) Brightfield and (G) fluorescent images of an exposed desmoid tumor (red arrowhead) with the skin partially removed showing active Wnt signaling in comparison to the neighboring exposed muscle (white arrows). The white arrowhead indicates autofluorescent skin overlying the tumor. 
neuroepithelial/neuroectodermal tumor (PNET). Areas of neuroepithelial differentiation were seen, with formation of primitive glandular structures resembling the neural tube and composed of pseudostratified, columnar-cuboidal epithelium (medulloepithelioma) (Fig. 4F).

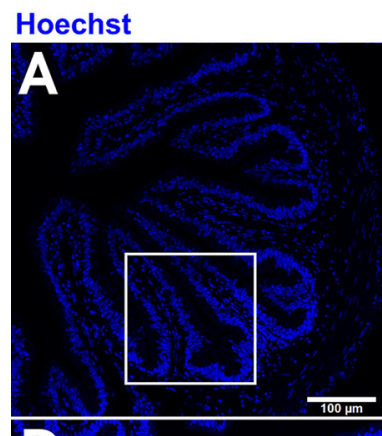

PCNA
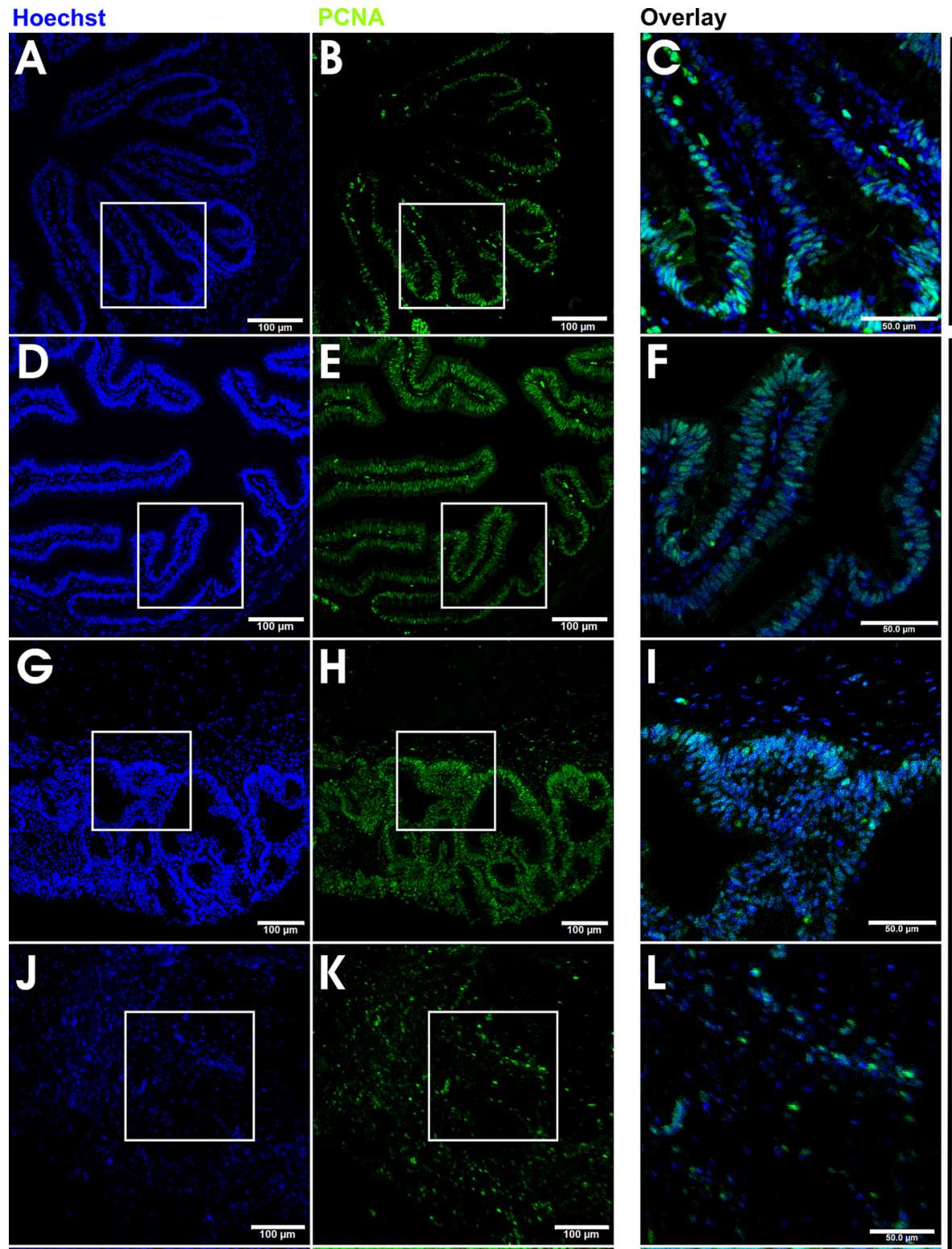

$100 \mathrm{pm}$
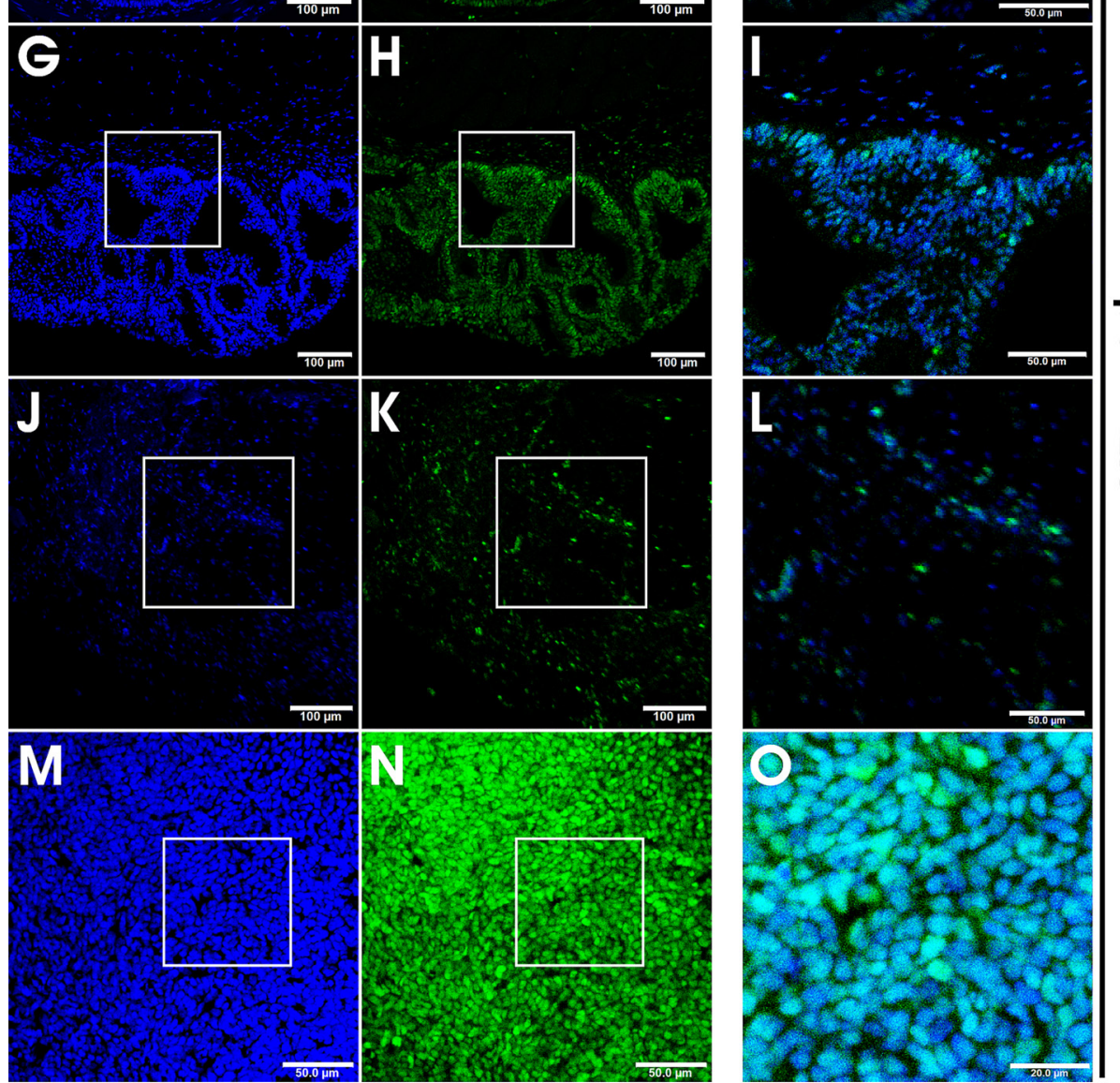

Figure 6: Increased and mislocalized proliferation in apc TALEN induced tumors. Hoechst (left panels) and PCNA (middle panels) double staining; magnified overlays in right panels. (A, B, C) In WT small intestine PCNA staining is primarily localized at the base of the folds and largely absent in the crests. (D, E, F) Small intestine of apc TALEN injected froglet showing the presence of PCNA staining along the entire trough-crest axis. (G, H, I) Epidermoid cysts showing a high number of proliferating PCNA positive cells. (J, K, L) Desmoid tumor displaying a large fraction of PCNA positive nuclei. (M, N, O) Hind limb-associated tumor with almost all nuclei staining positive for PCNA indicating a very high proliferation rate. 


\section{Medulloblastomas}

Some tadpoles injected with apc TALENs showed abnormal swimming behavior, including defects in balance. Abnormalities in the brain were apparent externally (Fig. 2F) or upon dissection. Histological examination of these tadpoles showed neoplasms that closely resemble medulloblastoma (Fig. 4G,H), which is another tumor type observed in a subset of FAP patients [22].

\section{Retinal hyperproliferation}

A fraction of tadpoles injected with the apc TALENs showed enlargement of the eyes. Histology showed abnormal organization of the retinal cell layers and the lens. The localized expansion of the cells of the neural retina adopted rosette-like structures and ectopically localized retinal pigment epithelium (Fig. 3C,D). These retinal malformations may be linked to Congenital Hypertrophy of the Retinal Pigment Epithelium (CHRPE), which is frequently observed in FAP patients [22].

\section{Wnt/ß-catenin signaling is active in apc mutant tumors}

To investigate whether the mosaic mutations in the MCR of apc resemble also functionally the situation in mammals and induce neoplasia with a hyperactive Wnt/ $\beta$-catenin signaling pathway, we injected the $a p c$ TALENs in embryos obtained from a transgenic $X$. tropicalis Wnt reporter line that we previously generated and characterized [23, 24]. GFP signals were analyzed in living tadpoles and in freshly dissected desmoid tumors. The most striking increase in GFP signal was observed in the fast-growing hind limb tumors (Fig. 5A-C) and in the epidermoid cysts (Fig. 5D,E). Remarkably, in the limb tumors the signal was especially strong in the peripheral region and was organized in undulated continuous strands

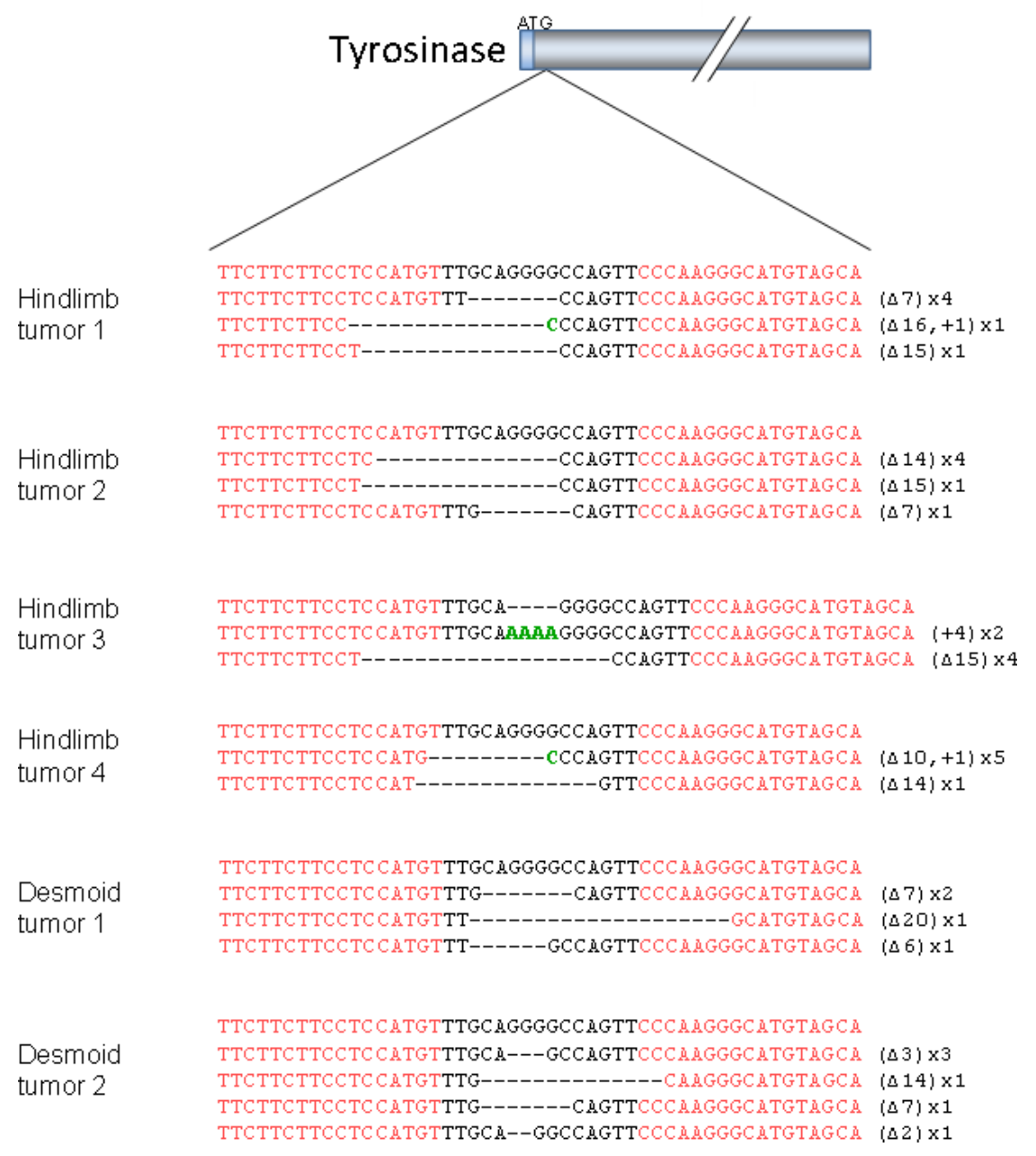

Figure 7: Combined bi-allelic targeting of the tyr and apc genes in $X$. tropicalis tumors. INDELs found in the tyr gene in apc mutant tumors developing after co-injection of apc (40 pg) and tyr TALEN (300 pg) mRNAs. Note that in contrast to the INDELs observed in the apc locus (see Fig. 1C), deletions in the tyr locus can be in frame, e.g. $\Delta 3$ or $\Delta 15$. 
(Fig. 5C). Histological examination and staining for phosphorylated histone 3 showed that the areas of highest GFP signal also had the highest cell density. It is unclear whether the intense GFP signal was due to a stronger Wnt response or primarily reflected the density of the cells in the tumor. Also the desmoid tumors showed increased GFP signals, primarily visible when the skin, which shows autofluorescence, was removed and the tumor was exposed (Fig. 5F,G). The fluorescent signal was weaker compared to the epidermoid cysts and the limb tumors but this is probably due to the fact that the fibromatous desmoid tumors are very rich in extracellular matrix but particularly low in cell density. Together, these data show that $\mathrm{Wnt} / \beta$-catenin signaling is increased, at least in the three types of tumors that were analyzed.

\section{apc mutant tumors show abnormal proliferation profiles}

To further examine whether TALEN-driven apc mutation affects cell proliferation, we performed proliferating cell nuclear antigen (PCNA) analysis on sections from intestines, epidermoid cysts, and the desmoid and hind limb tumors. As described previously [25], cell proliferation in the froglet intestine is restricted to the lower third of the trough-crest axis, which resembles the crypt-villus organization in mammals (Fig. 6A-C). In the intestines of frogs injected with apc TALEN, PCNA staining was uniform both in troughs and in crests of the longitudinal folds (Fig. 6D-F). This is reminiscent of the situation in mice after loss of $A p c$ and in humans carrying familial $A P C$ mutations [26-28].

Increased PCNA staining was also apparent in other tumor types that we analyzed by immunostaining, namely the epidermoid cysts (Fig. 6G-I) and desmoid tumors (Fig. $6 \mathrm{~J}-\mathrm{L})$. The limb tumor in particular showed rapid cell proliferation, with $>90 \%$ of the cells showing increased PCNA staining (Fig. 6M-O).

\section{Multiplexed gene disruption in Xenopus tumors}

A major asset of our model is the possible identification and characterization of genes that cooperate with APC-driven tumor formation or progression. To investigate whether we can obtain double bi-allelic gene disruptions in the tumors, the TALEN pair targeting the apc MCR was co-injected in two-cell stage embryos with a TALEN pair targeting the non-selective Tyrosinase (tyr) gene [29]. Tadpoles were grown until they developed limb and desmoid tumors. Tumor samples were obtained and DNA was extracted. The targeted region of the tyr gene was amplified by PCR and subcloned into pTOPO plasmids. All tumor samples contained only tyr alleles with INDEL mutations (Fig. 7), in addition to the obligate mutant $a p c$ alleles (data not shown). These results show that multiplexed bi-allelic gene targeting is straightforward in the developing Xenopus tumors.

\section{DISCUSSION}

We describe here a new and representative model for both familial and sporadic cancers associated with mutations in the $A P C$ tumor suppressor gene. Our Xenopus model develops at least three tumor types that are also found in human FAP patients: intestinal neoplasias, desmoid tumors, and medulloblastomas. Moreover, histological distortions in the Xenopus retina bear a resemblance to FAP-associated CHRPE. Two additional tumor types could not be linked to FAP, i.e. the limb tumors and the PNET-like outgrowths in the epidermis. These tumors might be development-related neoplasia, which are due to the early bi-allelic apc mutations that are inherent to the TALEN approach, and which do not occur in human FAP patients.

The vast majority of the Xenopus tumors contained only mutant $a p c$ alleles. For the epidermoid cysts, the desmoid tumors and the hind limb tumors we demonstrated increased activation of the Wnt/ $\beta$-catenin signaling pathway. Increased cell proliferation, as revealed by PCNA staining, was evident in all the tumor types. Interestingly, while the intestine was neoplastic, with proliferation extending throughout the crypt-villus axis, we did not observe genuine adenomas. We believe that this was due to the distinct histological organization of the post-metamorphic intestine of Xenopus, where cells are organized in continuous extended folds. This means that a clone of hyperproliferating cells would not be trapped locally, as would be the case in mammalian crypts. Instead, they can move sideways and cause expansion of the folds. This also explains the abnormal curly appearance of these folds (see Fig. 2B).

Cancer associated with $A P C$ mutations has already been extensively modeled in mice, both in spontaneous models such as the $\mathrm{Apc}^{\mathrm{Min}}$ mouse and in models using inducible tissue-specific $A p c$ truncations [3]. A zebrafish model using ENU-induced mutation of the Apc MCR region was also described [30]. Heterozygous mutant fish effectively developed adenomas associated with increased Wnt/ $\beta$-catenin signaling [4]. However, this only happened after 15 months in only a few of the adult animals [4]. Therefore, a faster aquatic model for APC-associated cancer with a higher penetrance, such as the Xenopus model described here, will be a major asset to the field.

Indeed, a major benefit of our model is the high penetrance and reproducibility of tumor formation and the rapid induction of the neoplasms, with the first desmoid tumors visible in six weeks. This opens the door for a myriad of applications including genotype-phenotype analysis by designing TALENs to different regions of the apc gene [31-33]. In addition, the rapid induction and reproducibility of tumor formation, combined with the 
aquatic habitat of the tadpoles and froglets, might permit fast and efficient pre-clinical screening of candidate compounds. It will be interesting to validate our model with experimental compounds or existing, clinically approved drugs, that were recently shown to interfere with APC associated intestinal or desmoid tumor formation [34-36].

In addition, we believe that our new Xenopus tumor model may be especially useful for identifying or characterizing possible effectors or modifiers of APC mediated tumor formation [37-39]. Also, analysis of tumor suppressors that cooperate with APC, or genes involved in tumor progression, would be a valuable extension of our model. The possibility of generating tumors with biallelic mutations of two independent genes -as shown here for the $a p c$ and the tyr genes- is an important benefit of our model. This approach is especially interesting for $X$. tropicalis, which is a tetrapod and a true diploid. In comparison, such approach would be less feasible in zebrafish, where about $30 \%$ of the genes are duplicated due to a genome duplication event that occurred during the phylogenetic development of the ray-finned fishes [40]. In addition, the very high synteny between the human and the $X$. tropicalis genomes facilitates the identification of the frog orthologs for most human genes of interest [14]. Evidently, Xenopus does not have the benefit of numerous existing mutant lines generated in zebrafish by the TILLING project and the Zebrafish Mutation project [10, 11]. However, considering current methods for efficient and cheap genome editing by using TALENs and CRISPR/ Cas9, this shortcoming is expected to be easily overcome.

In summary, we generated the first genuine genetic tumor model in $X$. tropicalis using TALEN-mediated targeting of the tumor suppressor gene $a p c$. Our approach would probably be feasible for other cancer-related genes, especially tumor suppressors. This should be an incentive for further use of this tetrapod organism for modeling human genetic diseases and cancer and as a model for pre-clinical drug screening. Xenopus models can become a powerful supplement to the currently frequently used murine and zebrafish models.

\section{MATERIALS AND METHODS}

\section{TALEN design, cloning and mRNA transcription}

A TALEN pair targeting the MCR of Xenopus tropicalis apc was designed using the TAL Effector Nucleotide Targeter 2.0 software [18], with spacer length and repeat array lengths all at $16 \mathrm{bp}$. The selected TALEN pair (Fig 1A) was cloned using the Golden Gate Cloning protocol [16], but using the pCS2-Flag-TALEN-ELD/ KKR vectors as the final backbones for the TALEN pair [41]. The TALEN pair targeting the tyr gene in $X$. tropicalis was a kind gift from Dr. Enrique Amaya [29]. These vectors were linearized using NotI, and mRNA was transcribed using the mMessage machine sp6 kit (Ambion). Finally, capped mRNA was extracted with phenol/chloroform, precipitated with isopropanol, and dissolved in nuclease-free water.

\section{Xenopus tropicalis microinjection}

Wild type and Wnt-reporter transgenic male and female $X$. tropicalis were primed 2 days before injection with 10 and $20 \mathrm{IU}$ of human chorionic gonadotropin (hCG), respectively. Natural matings were set up in the morning, after boosting males and females with 100 and $150 \mathrm{IU}$ of hCG, respectively. Embryos were collected and the jelly coat was removed using a $2 \%$ cysteine solution in 0.1X MMR (Marc's Modified Ringers solution). Embryos were injected unilaterally at the 2-cell stage with 40 or $300 \mathrm{pg} / \mathrm{embryo}$ of apc TALEN mRNA. At the 8-cell stage, single blastomeres (animal-ventral, animal-dorsal, vegetal-ventral or vegetal-dorsal) were injected with $20 \mathrm{pg}$ of apc TALEN mRNA. For the multiplexing experiment, tyr TALEN RNA and apc TALEN RNA were coinjected at a concentration of 300 and $40 \mathrm{pg} /$ embryo, respectively.

All experiments were performed in accordance with relevant guidelines and regulations of Ghent University, Faculty of Sciences.

\section{DNA extraction and sequencing}

Tadpoles and dissected tumors were incubated overnight at $55^{\circ} \mathrm{C}$ in lysis buffer $(50 \mathrm{mM}$ Tris $\mathrm{pH} 8.8$, $1 \mathrm{mM}$ EDTA, $0.5 \%$ Tween-20, $200 \mu \mathrm{g} / \mathrm{ml}$ proteinase $\mathrm{K})$. Genomic regions encompassing the apc and tyr TALEN cut sites were PCR amplified using specific primer pairs (apc: 5'-CATCCTAACTCTGCCCAA-3', 5'-ATAATGTTCTGGTGGGCT-3'; tyr: 5'-ACATATCAATCACCCCAACTC-3',

5'-TCTATCGTCAACCCCAGTG-3') and cloned using the pTOPO Zero Blunt ${ }^{\circledR}$ kit (Invitrogen). After transformation, plasmids were extracted from individual bacterial colonies and sequenced by the Sanger method.

\section{Imaging, histology and immunohistochemistry}

Living and dissected tadpoles and froglets were imaged on a Carl Zeiss StereoLUMAR.V12 stereomicroscope. Tissues and tumors were fixed in $4 \%$ PFA (paraformaldehyde), dehydrated, and embedded in paraffin. Sections of $5 \mu \mathrm{m}$ were rehydrated and stained with hematoxylin and eosin for histological examination. For PCNA immunohistochemistry, 5- $\mu \mathrm{m}$ sections were pressure cooked in citrate buffer $(10 \mathrm{mM}$ citric acid, $0.05 \%$ tween-20, $\mathrm{pH} 6$ ) for antigen retrieval and blocked 
in $3 \%$ goat serum, $1 \%$ BSA, $0.1 \%$ tween- 20 in PBS. Slides were incubated overnight with PCNA antibody (Clone PC10-Dako) at $4^{\circ} \mathrm{C}$. Secondary goat anti-mouse dylight-488 conjugated antibody was used for detection. Fluorescent tumor sections were imaged on a Leica TCS LSI zoom confocal microscope.

\section{ACKNOWLEDGEMENTS}

We would like to thank Prof. Dr. Em. Karel Geboes, consultant at the Department of Pathology at Ghent University Hospital for analyzing the histological intestinal sections. We are indebted to Sylviane Dewaele for technical assistance and Tim Deceuninck for animal care. The authors would like to thank Amin Bredan for critical reading and editing of the manuscript, and their colleagues for helpful discussions. The plasmids used in this study were obtained from Professor Christopher H.K. Cheng of School of Biomedical Sciences at the Chinese University of Hong Kong. Research in the authors' laboratory is supported by the Research Foundation Flanders (FWO-Vlaanderen), and by the Belgian Science Policy (Interuniversity Attraction Poles - IAP7/07). Further support was obtained by the Hercules Foundation, Flanders (grant AUGE/11/14). T.V.N. and T.N. holds a $\mathrm{PhD}$ fellowship with the agency for Innovation by Science and Technology (IWT).

\section{Author contribution}

K.V. and T.V.N. designed the study and wrote the manuscript, T.N., S.G., H.T.T. and G.V.I. were involved in TALEN design, embryo injections and immunostaining. E.S. and F.V.R. assisted in histological sections and stainings and D.C. performed the pathological analysis of the tissue sections.

\section{CONFLICT OF INTEREST}

The authors declare no conflict of interest

\section{REFERENCES}

1. Clark SK and Phillips RK. Desmoids in familial adenomatous polyposis. The British journal of surgery. 1996; 83(11):1494-1504.

2. Sturt NJ and Clark SK. Current ideas in desmoid tumours. Fam Cancer. 2006; 5(3):275-285; discussion 287-278.

3. Zeineldin $\mathrm{M}$ and Neufeld KL. More than two decades of Apc modeling in rodents. Biochimica et biophysica acta. 2013; 1836(1):80-89.

4. Haramis AP, Hurlstone A, van der Velden Y, Begthel H, van den Born M, Offerhaus GJ and Clevers HC. Adenomatous polyposis coli-deficient zebrafish are susceptible to digestive tract neoplasia. EMBO Rep. 2006; 7(4):444-449.

5. Irving AA, Yoshimi K, Hart ML, Parker T, Clipson L, Ford MR, Kuramoto T, Dove WF and Amos-Landgraf JM. The utility of Apc-mutant rats in modeling human colon cancer. Disease models \& mechanisms. 2014; 7(11):1215-1225.

6. Hahne G and Grossmann TN. Direct targeting of betacatenin: Inhibition of protein-protein interactions for the inactivation of Wnt signaling. Bioorganic \& medicinal chemistry. 2013; 21(14):4020-4026.

7. Zimmerman ZF, Moon RT and Chien AJ. Targeting Wnt pathways in disease. Cold Spring Harb Perspect Biol. 2012; 4(11).

8. Kahn M. Can we safely target the WNT pathway? Nat Rev Drug Discov. 2014; 13(7):513-532.

9. Schmitt SM, Gull $M$ and Brandli AW. Engineering Xenopus embryos for phenotypic drug discovery screening. Advanced drug delivery reviews. 2014; 69-70:225-246.

10. Kettleborough RN, Busch-Nentwich EM, Harvey SA, Dooley CM, de Bruijn E, van Eeden F, Sealy I, White RJ, Herd C, Nijman IJ, Fenyes F, Mehroke S, Scahill C, Gibbons R, Wali N, Carruthers S, et al. A systematic genome-wide analysis of zebrafish protein-coding gene function. Nature. 2013; 496(7446):494-497.

11. Moens CB, Donn TM, Wolf-Saxon ER and Ma TP. Reverse genetics in zebrafish by TILLING. Brief Funct Genomic Proteomic. 2008; 7(6):454-459.

12. White R, Rose K and Zon L. Zebrafish cancer: the state of the art and the path forward. Nature reviews Cancer. 2013; 13(9):624-636.

13. Peng Y, Clark KJ, Campbell JM, Panetta MR, Guo Y and Ekker SC. Making designer mutants in model organisms. Development. 2014; 141(21):4042-4054.

14. Hellsten U, Harland RM, Gilchrist MJ, Hendrix D, Jurka J, Kapitonov V, Ovcharenko I, Putnam NH, Shu S, Taher L, Blitz IL, Blumberg B, Dichmann DS, Dubchak I, Amaya E, Detter JC, et al. The genome of the Western clawed frog Xenopus tropicalis. Science. 2010; 328(5978):633-636.

15. van Es JH, Giles RH and Clevers HC. The many faces of the tumor suppressor gene APC. Exp Cell Res. 2001; 264(1):126-134.

16. Cermak T, Doyle EL, Christian M, Wang L, Zhang Y, Schmidt C, Baller JA, Somia NV, Bogdanove AJ and Voytas DF. Efficient design and assembly of custom TALEN and other TAL effector-based constructs for DNA targeting. Nucleic acids research. 2011; 39(12):e82.

17. Doyon Y, Vo TD, Mendel MC, Greenberg SG, Wang J, Xia DF, Miller JC, Urnov FD, Gregory PD and Holmes MC. Enhancing zinc-finger-nuclease activity with improved obligate heterodimeric architectures. Nat Methods. 2010; 8(1):74-79.

18. Doyle EL, Booher NJ, Standage DS, Voytas DF, Brendel VP, Vandyk JK and Bogdanove AJ. TAL EffectorNucleotide Targeter (TALE-NT) 2.0: tools for TAL effector design and target prediction. Nucleic acids research. 2012; 
40(Web Server issue):W117-122.

19. Miyoshi $Y$, Nagase H, Ando H, Horii A, Ichii S, Nakatsuru S, Aoki T, Miki Y, Mori T and Nakamura Y. Somatic mutations of the APC gene in colorectal tumors: mutation cluster region in the APC gene. Hum Mol Genet. 1992; 1(4):229-233.

20. Moody SA. Fates of the blastomeres of the 16-cell stage Xenopus embryo. Developmental biology. 1987; 119(2):560-578.

21. Sun G, Hasebe T, Fujimoto K, Lu R, Fu L, Matsuda H, Kajita M, Ishizuya-Oka A and Shi YB. Spatio-temporal expression profile of stem cell-associated gene LGR5 in the intestine during thyroid hormone-dependent metamorphosis in Xenopus laevis. PloS one. 2010; 5(10):e13605.

22. Half E, Bercovich D and Rozen P. Familial adenomatous polyposis. Orphanet J Rare Dis. 2009; 4:22.

23. Tran HT, Sekkali B, Van Imschoot G, Janssens $\mathrm{S}$ and Vleminckx K. Wnt/beta-catenin signaling is involved in the induction and maintenance of primitive hematopoiesis in the vertebrate embryo. Proceedings of the National Academy of Sciences of the United States of America. 2010; 107(37):16160-16165.

24. Tran HT and Vleminckx K. Design and use of transgenic reporter strains for detecting activity of signaling pathways in Xenopus. Methods. 2013; 66(3):422-432.

25. Schreiber AM, Cai L and Brown DD. Remodeling of the intestine during metamorphosis of Xenopus laevis. Proceedings of the National Academy of Sciences of the United States of America. 2005; 102(10):3720-3725.

26. Santucci R, Volpe L, Zannoni U, Paganelli GM, Poggi B, Calabrese $\mathrm{C}$ and Biasco G. Cell proliferation of the duodenal mucosa in patients affected by familial adenomatous polyposis. Gastroenterology. 1997; 113(4):1159-1162.

27. Polyak K, Hamilton SR, Vogelstein B and Kinzler KW. Early alteration of cell-cycle-regulated gene expression in colorectal neoplasia. Am J Pathol. 1996; 149(2):381-387.

28. Yoshikawa R, Hatada T, Kusunoki M, Yamamura T and Utsunomiya J. Assessment of cell-proliferation kinetics in familial adenomatous polyposis by pena immunostaining. Int J Oncol. 1993; 3(2):341-345.

29. Ishibashi S, Cliffe R and Amaya E. Highly efficient biallelic mutation rates using TALENs in Xenopus tropicalis. Biology open. 2012; 1(12):1273-1276.

30. Hurlstone AF, Haramis AP, Wienholds E, Begthel H, Korving J, Van Eeden F, Cuppen E, Zivkovic D, Plasterk $\mathrm{RH}$ and Clevers $\mathrm{H}$. The Wnt/beta-catenin pathway regulates cardiac valve formation. Nature. 2003; 425(6958):633-637.

31. Fodde R and Khan PM. Genotype-phenotype correlations at the adenomatous polyposis coli (APC) gene. Critical reviews in oncogenesis. 1995; 6(3-6):291-303.

32. Heinen CD. Genotype to phenotype: analyzing the effects of inherited mutations in colorectal cancer families. Mutation research. 2010; 693(1-2):32-45.

33. Nieuwenhuis MH and Vasen HF. Correlations between mutation site in APC and phenotype of familial adenomatous polyposis (FAP): a review of the literature. Critical reviews in oncology/hematology. 2007; 61(2):153161.

34. Faller WJ, Jackson TJ, Knight JR, Ridgway RA, Jamieson T, Karim SA, Jones C, Radulescu S, Huels DJ, Myant KB, Dudek KM, Casey HA, Scopelliti A, Cordero JB, Vidal M, Pende M, et al. mTORC1-mediated translational elongation limits intestinal tumour initiation and growth. Nature. 2014.

35. Ghanbari-Azarnier R, Sato S, Wei Q, Al-Jazrawe M and Alman BA. Targeting stem cell behavior in desmoid tumors (aggressive fibromatosis) by inhibiting hedgehog signaling. Neoplasia. 2013; 15(7):712-719.

36. Valvezan AJ, Huang J, Lengner CJ, Pack M and Klein PS. Oncogenic mutations in adenomatous polyposis coli (Apc) activate mechanistic target of rapamycin complex 1 (mTORC1) in mice and zebrafish. Disease models \& mechanisms. 2013; 7(1):63-71.

37. Cathcart MC, O’Byrne KJ, Reynolds JV, O'Sullivan J and Pidgeon GP. COX-derived prostanoid pathways in gastrointestinal cancer development and progression: novel targets for prevention and intervention. Biochimica et biophysica acta. 2012; 1825(1):49-63.

38. Kwong LN and Dove WF. APC and its modifiers in colon cancer. Advances in experimental medicine and biology. 2009; 656:85-106.

39. McCart AE, Vickaryous NK and Silver A. Apc mice: models, modifiers and mutants. Pathology, research and practice. 2008; 204(7):479-490

40. Hoegg S, Brinkmann H, Taylor JS and Meyer A. Phylogenetic timing of the fish-specific genome duplication correlates with the diversification of teleost fish. J Mol Evol. 2004; 59(2):190-203.

41. Lei Y, Guo X, Liu Y, Cao Y, Deng Y, Chen X, Cheng $\mathrm{CH}$, Dawid IB, Chen Y and Zhao H. Efficient targeted gene disruption in Xenopus embryos using engineered transcription activator-like effector nucleases (TALENs). Proceedings of the National Academy of Sciences of the United States of America. 2012; 109(43):17484-17489. 